\title{
Pathohistological Aspects of Inflammatory Cysts
}

\author{
Oliver Dimitrovski ${ }^{1}$, Vanco Spirov ${ }^{2}$, Blagoja Dashtevski ${ }^{3}$ \\ ${ }^{1}$ University Sts Cyril and Methodius in Skopje, Faculty of Dentistry Clinic for Oral Surgery, Vodnjanska 19, Skopje, R. Macedonia \\ ${ }^{2}$ University Sts Cyril and Methodius in Skopje, Faculty of Dentistry Clinic for Oral Surgery, Vodnjanska 19, Skopje, R. Macedonia
}

${ }^{3}$ University Sts Cyril and Methodius in Skopje, Faculty of Dentistry, Clinic for Prosthetic Dentistry, Vodnjanska 19, Skopje, R. Macedonia

\begin{abstract}
Undefined etiopathogenetic mechanism of inflammatory cysts and frequent complications were the subject of our interest. The study was focused on pathohistological examination of inflammatory cysts and possible effect on the local etiogenetic factors in the evolution and prognosis of the cystic lesion. Material and methods: A total of 150 patients with a diagnosis of inflammatory cysts were examined. All of the patients were assigned to 3 groups, each comprising 50 patients according to the diagnosis: group $A$-patients with radicular cysts; $B$ - patients with residual cysts and group $C$-patients with periodontal cysts. The control group consisted of 35 patients without cystic disorders. Clinical examination was based on anamnesis and clinical data. Paraclinical segment involved patohistological evidence. Results: Pathohistological aspect showed predominantly chronic exacerbation infiltrate (radicular 56\%, residual $64 \%$ and periodontal 66\%), versus common chronic infiltrate. Regarding epithelium, sheet squared epithelium was predominating (72\% in radicular, $66 \%$ in residual and $74 \%$ in periodontal cysts) while cylindrical epithelium was very rare. Conclusions: Our results provide concrete evidence about the nature of the inflammatory cysts (etiopathogenesis of this frequently present oral cavity disorder).
\end{abstract}

Keywords: cysts of the jaw, inflammatory cysts, radicular cyst, residual cyst, periodontal cysts

\section{Introduction}

Cysts which appear in the orofacial region are common pathological conditions that often end up with complications. In the past, the appearance and diagnosis of the cysts presented a great difficulty. Nowadays, in spite of the great progress and development of science and technology, the diagnosis of the cysts, especially their differentiation is still a serious obstacle which is not easy to be overcome. Cysts are often found in the clinical practice. There has been an increase of these changes mainly due to the severity of the signs and symptoms but also due to many other reasons. Some of them are: complications due to untimely and inadequate treatment, harmful effect on the general health condition; they might be potential focus of infection which affect the other systems and organs.

The specific development, regardless the growth and complexity of the condition in the cystic lining have been a subject of a large number of investigations [1], [2].

The diagnosis of the cysts is not a complex issue since there are a large number of clinical and para-clinical methods which yield satisfactory results. Nevertheless, in many conditions when there are not enough parameters in order to establish a solid diagnosis, then histopathological verification is necessary [3].

Cysts are sac-like structures composed of connective tissue which inside surface is coated with epithelial membrane. The inside epithelial layer limits its inner space making close contact with the cystic contents. The outer layer overlaps the surrounding bone. Thus, a close link between different tissues is made, and it is not only mechanical but also humoral where a lot of immunopathologic events happen. The epithelial layer of the bone cysts is the basic histopathological characteristic of this entity. Its characteristics (type, thickness, homogeneity) depend mainly on the type of the cyst as well as on the degree of inflammatory changes in the cyst walls.

The type of the epithelium is not a pathognomic characteristic which determines the cyst type. Thus, in the group of ontogenetic cysts, the epithelial layer is made of multilayer epithelial lining. Unlike them, the radicular cysts on the maxilla which have contact with mucosa of the maxillary sinus or the nose have pseudo layer epithelium. On the mandible, this type of epithelium that can be found in the radicular cysts is very rare. In the radicular cysts, in addition to multilayer epithelial lining, tubular epithelium can also be found.

Measuring the activity of the collagenase by monitoring the specific degradation products, type 1 and 2 collagen in SDS - PAGE solution, shows that all cyst extracts contain collagenase, and some of them are endogenously activated. This way, the cyst wall of the collagenase degrades mainly in type 1 collagen, which suggests that the degradation is mmp-1 (Matrix metalloproteinase- 1). This finding is supported by doxycycline inhibition of cystic collagenase which is similar to mmp-1 [4].

The thickness of the epithelial layer is different and usually the developing cysts are characterized by a thin layer of epithelium made of three to four layers of cells. Sometimes the long-term persisting radicular cysts which do not have acute exacerbation can have epithelial layer that has several layers of epithelial cells with palisade positioned basal cells.

Hyaline cells with different structure can be noticed in the epithelium of some cysts of dental origin. They were discovered for the first time by Rushton and are named Rushton cells. A characteristic of some dental cysts are cholesterol crystals in cystic cover.

Etiopathogenetic mechanisms of the inflammatory cysts are still not well defined, as well as the common complications that occur if they are present in the mouth. This was the 


\section{International Journal of Science and Research (IJSR) \\ ISSN (Online): 2319-7064}

Index Copernicus Value (2013): 6.14 | Impact Factor (2014): 5.611

main motive for this study - to analyze the pathohistologic characteristics of the inflammatory cysts, their structure and how the inflammation affects the appearance, development and changes of this type of cysts and also to determine the possible similarities and differences between radicular, residual and periodontal cysts from pathohistological aspect.

\section{Material and Method}

In the period between 2004 and 2007, a total of 150 patients at the age between $18-60$ years, of both sexes, with clinically diagnosed inflammatory cysts were studied. All patients that were included in this study were clinically followed-up at the Clinic for Oral Surgery and the Dental Clinical Centre St. Panteleimon in Skopje. All of the patients signed an informed consent to participate in the study and the study was approved by the Ethics Commission. Some of the anticipated analyses were conducted at the Institute of Pathology at the Medical Faculty in Skopje. In order to achieve the goals of the study, the participants were divided in two groups: examined group of 150 participants and control group of 35 participants.

The pathohistological analyses of the cystic changes were done on bioptic material using the Haematoxylin method (HE), Eosin (mastocyti Eo), Van Gieson. The material taken from the lesion was put in a sterile syringe filled with physiological solution, and transported to the Institute of Pathology, where the material was placed in $10 \%$ formalin (formaldehyde). The tissue was fixed for 24 hours, and then analyzed in tissue processor or autotechnical SHANDON (Germany) model CITADEL 2000. Later, the material was molded in paraffin and was cut with microtome $5 \mu$ thick. Then the procedure continued with dyeing the material using a standard histochemical procedure (dyeing) by Haematoxylin, Eosin and Van Gieson. The glass along with the material were covered with Kanada balsam and then with a cover slip. The material was analyzed under light microscope - Leitz Wetzlar (Germany) model Dialux 20, which enlarges $40-400$ times. The results obtained from the clinical and other analyses of the examined and the control group were processed with analytical and descriptive procedures i.e. methods.

The following descriptive methods were used: percentage structure, average amount - arithmetic mean (X), standard deviation (SD), standard error (SE), mode (Mo). The following analytic methods were used: $\mathrm{t}-$ test, $\mathrm{X}^{2}-$ test, analysis of variance, Turkey's post hoc test.

\section{Results}

The pathohistological examination of the inflammatory infiltrate, eosinophil, epithelium and the granulation tissue has given the following results. Regarding the infiltrate, inside the examined groups, a large percentage of absence of chronic exacerbate infiltrate was noticed $-56.0 \%, 64.0 \%$ and $66.0 \%$ (fig. 1). The obtained findings in our study have shown that all three types of cysts (radicular, residual and periodontal) have predominant chronic exacerbate infiltrate against the ordinary chronic infiltrate. The proportional presentation of the infiltrate in the radicular cysts was 56.6
$\%$, in the residual cysts $64 \%$, and in the periodontal cysts $66 \%$.

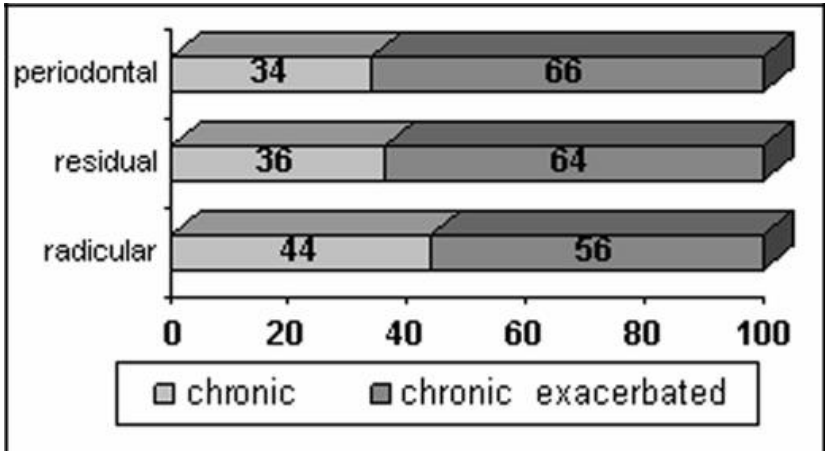

Figure 1: Percentage difference between the three studied groups of inflammatory cysts of the jaw

The radicular cyst wall, which is covered with multilayer epithelial lining under which a collagen connective tissue can be found, is shown in Figure 2.

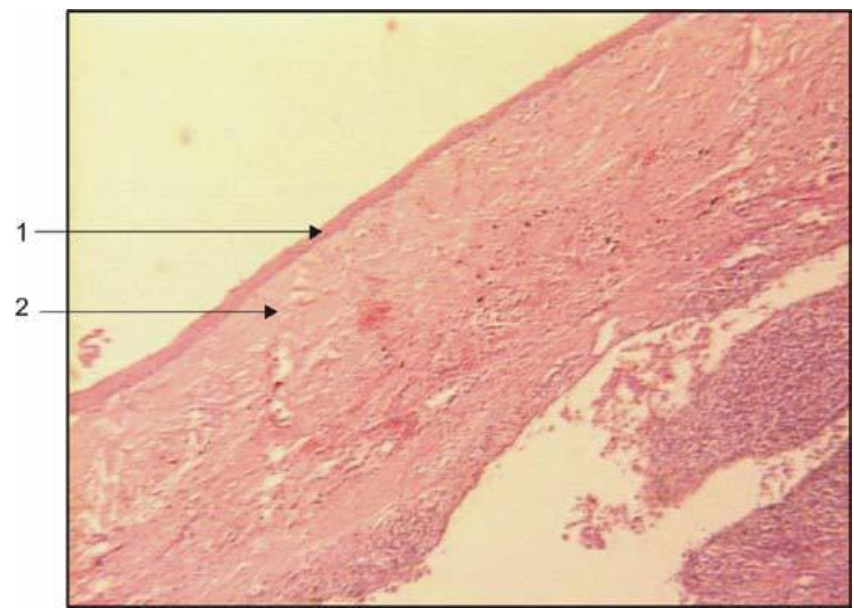

Figure 2: Pathohistological preparation of radicular cyst's wall: (1) multilayer epithelial lining; (2) connective collagen tissue. (increase - h40; dyeing - HE)

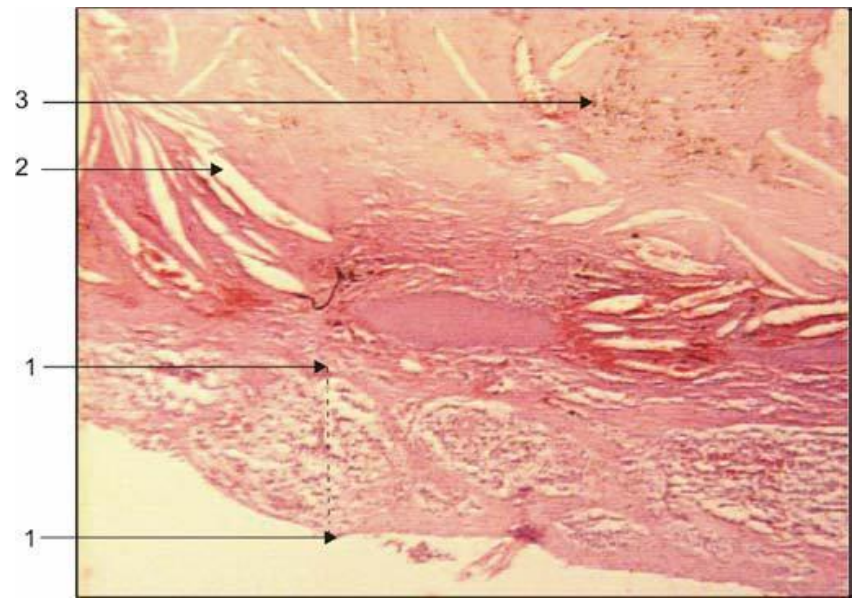

Figure 3: Pathohistological preparation of radicular cyst's wall: (1) granular tissue; (2) cholesterol crystals; (3) hemosiderin pigment (increase - h40; dyeing - HE);

In the epithelial lining a desquamated epithelium was evident, under which a granulation tissue was found and 


\section{International Journal of Science and Research (IJSR) \\ ISSN (Online): 2319-7064}

Index Copernicus Value (2013): 6.14 | Impact Factor (2014): 5.611

under that there were cholesterol crystals as well as hemosiderin pigmentation (Fig. 3 ).

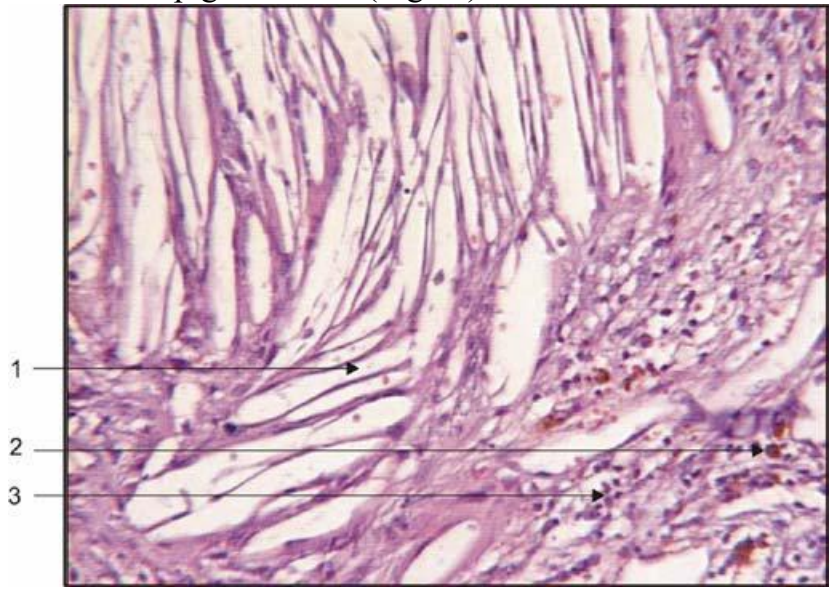

Figure 4: Pathohistological preparation of radicular cyst's wall: (1) cholesterol crystals; (2) hemosiderin pigment alone and in macrophages; (3) inflammatory infiltrate (increase h200; dyeing - HE)

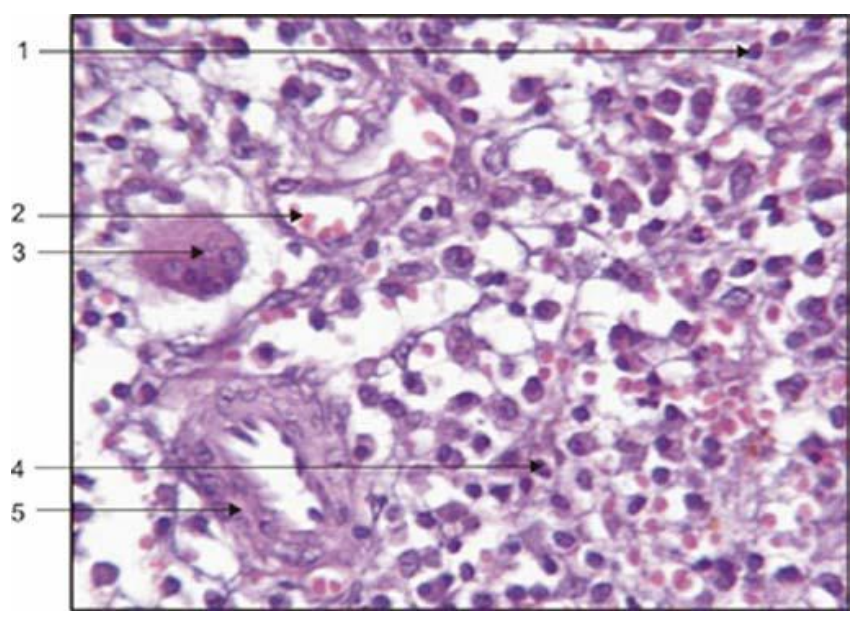

Figure 5: Pathohistological preparation of radicular cyst's wall: (1) plasma cells; (2) venules; (3) giant foreign cells;

(4) neutrophils; (5) arteriole (increase - h400; dyeing - HE)

Numerous cholesterol crystals could also be found, as well as inflammatory infiltrate of chronic type and intracellular and extracellular hemosiderin pigmentation (Fig.4 ). Figure 5 illustrates chronic inflammatory infiltrate mainly consisting of plasma cells and lymphocytes, some neutrophils and foreign body giant cells.

The percentage difference between the three examined groups regarding the pathohistological finding - chronic infiltrate and chronic exacerbate infiltrate was statistically insignificant $(\mathrm{p}>0.05 ; \mathrm{p}=0.3729, \mathrm{p}=0.2637$ and $\mathrm{p}=0.8187)$ (fig. 1).

In a larger percentage $(72.0 \%, 78.0 \%$ and $80.0 \%)$ of the examined groups there were no eosinophils. The different percentage in the three examined groups regarding the presence of eosinophils was statistically significant $\mathrm{p}=0.0000$ (Fig. 6).

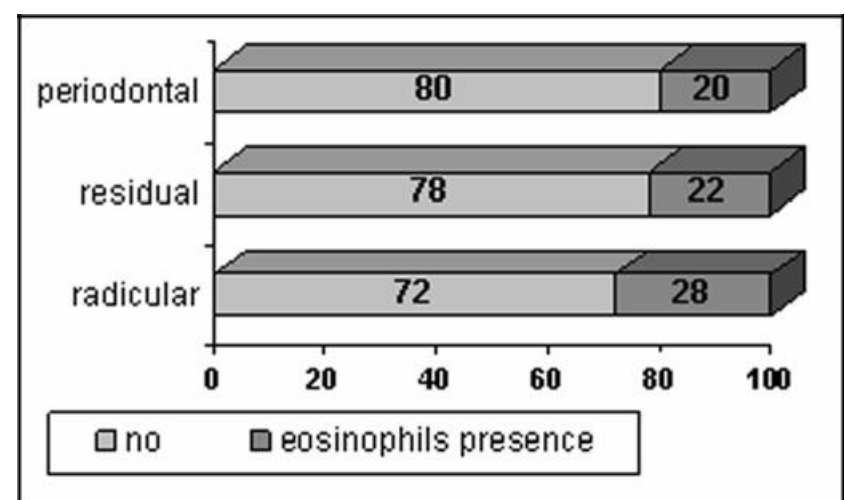

Figure 6: Percentage difference between the three studied groups of inflammatory cysts of the jaw regarding presence of eosinophils

Table 1: Epithelial lining type regarding to inflammatory cysts of the jaw

\begin{tabular}{|c|c|}
\hline Epithelial Lining & $\mathrm{N}^{\mathrm{o}} / \%$ \\
\hline Radicular cysts & \\
\hline No presence & $4 / 8.0$ \\
\hline Plate epithelium & $36 / 72.0$ \\
\hline Cylindric epithelium & $9 / 18.0$ \\
\hline Other epithelium types & $1 / 2$ \\
\hline Residual cysts & \\
\hline No presence No presence & $6 / 12$ \\
\hline Plate epithelium & $33 / 66$ \\
\hline Cylindric epithelium & $5 / 10$ \\
\hline Other epithelium types & $4 / 8$ \\
\hline Plate and cylindric epithelium & $2 / 4.0$ \\
\hline Periodontal cysts & \\
\hline No presence & $11 / 22$ \\
\hline Plate epithelium & $32 / 64$ \\
\hline Cylindric epithelium & $2 / 4.0$ \\
\hline Other epithelium types & $3 / 6.0$ \\
\hline Plate and cylindric epithelium & $2 / 4.0$ \\
\hline
\end{tabular}

The type of the epithelium layer of the radicular, residual and periodontal cysts was histologically determined. With no doubt, all participants had a predominant epithelial lining i.e. out of 50 cases of radicular cysts it was found in 36 $(72 \%)$, in the residual in $33(66 \%)$, in the periodontal in 32 $(64 \%)$. On the other hand, the tubular epithelium was present in a smaller number of the examined cases.

The radicular cysts are wrapped with this type in only 9 cases $(18 \%)$, the residual in 5 cases $(10 \%)$ and the periodontal in only 2 cases $(4 \%)$. There was no cystic epithelium in 11 cases $(22 \%)$ of the periodontal cysts, in 6 $(12 \%)$ of the residual cysts, and in $4(8 \%)$ of the radicular cysts.

The largest percentage of epithelial lining was registered in the three examined groups $(72 \%, 66 \%$ and $74 \%)$. The finding was statistically significant $-\mathrm{p}=0.0000$ (Tab. 1). The difference in the percentage between the groups with radicular and residual cysts regarding the presence of tubular epithelium was statistically insignificant $(p=0.2091)$, and that between the radicular and periodontal cysts was statistically significant $(p=0.0157)$. The percentage difference between the groups of residual and periodontal cysts regarding the presence of tubular epithelium was 


\section{International Journal of Science and Research (IJSR) \\ ISSN (Online): 2319-7064}

Index Copernicus Value (2013): 6.14 | Impact Factor (2014): 5.611

statistically insignificant $(p=0.2003), \quad($ graph 3$)$. The percentage difference between the three examined groups regarding the presence of other type of epithelium was statistically insignificant $(\mathrm{p}>0.05 ; \mathrm{p}=0.1343, \mathrm{p}=0.2658$ and $\mathrm{p}=0.6685)$; (Tab. 1).

Regarding the presence of granulation tissue in the three examined groups, it was observed that the granulation tissue in the cystic lesion was most common in the radicular cysts $-43(86 \%)$ of the total number of participants. The residual and periodontal cysts were found in a very similar i.e. identical percentage. Namely, the radicular cysts had granulation tissue in $41(82 \%)$ cystic lesions and the residual in $40(80 \%)$. The absence of granulation tissue was smallest in the radicular cysts whereas there was almost no difference between the residual and periodontal cysts.

No granulation tissue was registered in the largest percentage of the examined groups $(86 \%, 82 \%$ and $80 \%)$; the finding was statistically significant $-\mathrm{p}=0.0000$ (fig. 7 ).

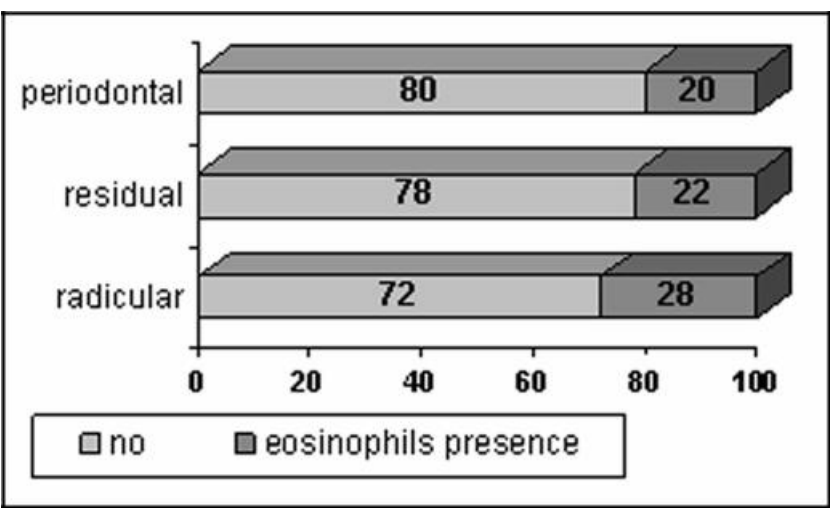

Figure 7: Percentage difference between the three studied groups of inflammatory cysts of the jaw regarding presence of granulation tissue

\section{Discussion}

The clinical and radiological methods are the primary methodological procedures in diagnosing the inflammatory cysts. But in the largest number of cases they are not enough for definitive and exact diagnosis. Actually the precise nature of the cystic formation (residual, periodontal and radicular) is most precisely defined by using histological methods. Final precision is obtained by conducting histological analysis of the extirpated changes, which greatly contributes to establishing the correct diagnosis. These findings comply with the findings presented by Ricucci et al. $[5]$.

Moreover, by using the histological preparations with additional monoclonal dyeing one can go deeper into mechanisms which in a way induce the appearance and evolution of the cysts. There is a connection between the mucous and vacuolated cells in the epithelial layer of the radicular and residual cysts and the vacuolar cells can represent a stage in the histogenesis in the mucosal metaplasia of these layers [6]. The mucosal cells are present separately or in groups in all layers of the layered squamous epithelium, except in the basal cell layers. Almost all cases have small to large ovoid vacuolar cells which are closely associated to the mucous cells. Sometimes the vacuolar cells contain mucin granules or diastase - resistant material. It is suggested that the empty cells can represent a stage in the histogenesis of the mucosal metaplasia. The findings of our study have shown that all three types of cysts (residual, periodontal and radicular) had a predominating chronic exacerbate infiltrate against the ordinary chronic infiltrate. The registered infiltrate in the three examined types of cystic lesions undoubtedly indicates that the processes which occur inside the cystic lesion have chronic exacerbate infiltrate. These facts speak in favor of the clinical finding of each cyst, which confirms the established diagnosis.

The different percentage in the two infiltrates in the groups with residual and periodontal cysts was statistically significant $(\mathrm{p}=0.0062$ and $\mathrm{p}=0.0019)$, while in the group with radicular cysts it was statistically insignificant $(p=0.2333)$, which we assume was due to our random samples. We also think that these results apply to the large incidence of exacerbation of the inflammatory cystic changes especially in conditions of reduced patients' immunity and present etiological hazards. Our results are in accordance with Sun and Liao's findings [7].

The maxillary radicular cysts have pseudolayer epithelium because they have contact with the mucous membrane of the maxillary sinus or the nose. This type of epithelium which can be found in the radicular cysts is very rare on the mandible. The radicular cysts, in addition to the multilayer epithelial lining can also have tubular epithelium.

Our analysis of the contents of all types of cystic lesions regarding the presence of eosinophils indicated that they were not present in sufficient number.

In the radicular cysts, the eosinophils were present in 14 cases $(28 \%)$ out of 50 participants, in the residual in 11 $(22 \%)$ and in the periodontal in 10 cases $(20 \%)$. Taken into consideration that all three types of examined cysts in more than $70 \%$ of the examined content did not contain eosinophils, we assume that the allergic component is not the dominant part of the etiopathogenesis of this common changes in the mouth. The minimal finding of eosinophils is considered as a coincidence in our cases.

We assume that the percentage difference between the three examined groups regarding eosinophils presence which was statistically insignificant $(\mathrm{p}>0.05 ; \mathrm{p}=0.4494, \mathrm{p}=0.3070$ and $\mathrm{p}=0.7884$ ), (graph 2) was due to our random samples.

The epithelium structure depends on the presence and amount of inflammatory changes in the subepithelium and connective tissue space. The inflammatory cysts, which have a high degree of subepithelial chronic inflammation, have significant changes in the continuity of the epithelium. The epithelial discontinuity is manifested with changes in its structure where microscopically observed, epithelial dispersion is noticed. This is a consequence of weakening and lysis of the intercellular desmosomal junctions in the inflammatory processes. In some cases, in cases of extremely expressed inflammation with secondary infection, necrotic zones can be noticed where a loss of continuity or total epithelium necrosis can be found. 


\section{International Journal of Science and Research (IJSR) \\ ISSN (Online): 2319-7064}

Index Copernicus Value (2013): 6.14 | Impact Factor (2014): 5.611

The percentage difference between the three examined groups regarding presence of epithelial lining was statistically insignificant $(\mathrm{p}>0.05 ; \mathrm{p}=0.4788, \mathrm{p}=0.3495$ and $\mathrm{p}=0.8187$ ) (graph 3 ) as well as the difference between the groups of radicular and residual cysts regarding the presence of tubular epithelium $(p=0.2091)$. We think that these differences are due to our random samples/randomly selected samples.

We assume that the epithelium enters the cystic capsule that is made of granular tissue infiltrated with a mixture of chronic inflammatory cells. The found infiltrate does not have equal intensity on the whole surface of the cystic capsule. Moving from the center to the periphery the mature fibrous tissue is gradually reduced and replaced by granulation tissue. A characteristic of some denture cysts are cholesterol crystals which are found in the cystic lining. Their origin is not completely explained but it is considered that they can be a result of accumulation of the circular plasma lipids found in people with expressed arteriosclerosis. Thus, there is a correlation of their presence and the findings of macrophages with hemosiderin. The presence of Rushton's bodies in $10 \%$ of the cases is also significant [8].

These hyaline formations are rarely found in the connective tissue layer and are most commonly localized in the epithelium which has amorphous structure on these places. Their origin is not yet clarified, but it is considered that they originate from the thrombus of the small blood vessels [9]. The origin of the cholesterol crystals is not completely explained but it is thought that they could be a result of accumulation of circulating plasma lipids found in people with expressed arteriosclerosis [10]. The histological preparations clearly show cholesterol crystals most commonly localized in the connective tissue layer near the giant cells that point out to a reaction of foreign bodies.. The connective tissue layer is incorporated in the thicker peripheral layer of the cystic formations which consists of collagen fibers with different structure and appearance which are directly dependent on the amount of inflammatory changes. In contrast, in our study, we did not notice presence of cholesterol crystals in the residual cysts, and the layers and the capsule were similar to the radicular - they were more mature, with tendency of the epithelium to extend inside the capsule.

Our findings are similar to those presented by Gordeeff [3] and Teronen [9], but opposite to those of Yamamoto [8].

The percentage difference between the three examined groups regarding the presence of granulation tissue was statistically insignificant $(\mathrm{p}>0.05 ; \mathrm{p}=0.5512, \mathrm{p}=0.3834$ and $\mathrm{p}=0.7806$ ); (graph 4), which was due to our random samples.

\section{Conclusions}

In conclusion, we assume that inflammation changes the morphology of the cyst's epithelium by changing its consistency and density. The smaller inflammatory component leads to disintegration of the epithelial tissue and decrease of its density.
On the other hand, a very strong inflammation or infection leads to epithelium changes i.e. its partial or total necrosis or its total loss. In cases where there is absence of inflammation the epithelium shows tendency of decreasing its volume and there is no presence of sub-basal proliferations, but there is stressed hyalinization that is manifested with appearance of hyaline bodies. The epithelial layer can show presence of mucous or cylindrical cells, which we assume happens in largest percentage when cystic lesions are extended toward the maxillary sinus or the nasal canal where this type of epithelium originates.

\section{References}

[1] A. Benn, M. Altini," Dentigerous cysts of inflammatory origin. A clinicopatho-ogic study". Oral Surgery Oral Medicine Oral Pathology Oral Radiology Endodontics, Feb; (2):pp03-9, 1996.

[2] R.S. Redman, "Respiratory epithelium in an apical periodontal cyst of the mandible". Oral Surgery Oral Medicine Oral Pathology Oral Radioogy Jan;67(1): pp77-80, 1989.

[3] M. Gordeeff, S. Clergeau-Guerithault, A. Gordeeff, "Histopathology of odontogenic cysts", Journale de Biologie Buccale, Sep;17(3):pp131-46, 1989.

[4] O. Teronen, T. Salo, J. Laitinen, J. Tornwall, P. Ylipaavalniemi, Y.T. Konttinen, J. Hietanen, T. Sorsa. "Characterization of interstitial collagenases in jaw cyst wall”. European Journal of Oral Science, Jun;103(3): pp141-7, 1995.

[5] D. Ricucci, E.A. Pascon, T.R. Ford, K. Langeland, "Epithelium and bacteria in periapical lesions". Oral Surg Oral Surgery Oral Medicine Oral Pathology Oral Radiology Endodontics, Aug; 102(2): 142, 2006.

[6] H. Slabbert, M. Shear, M. Altini, "Vacuolated cells and mucous metaplasia in the epithelial linings of radicular and residual cysts", Journal of Oral Pathology and Medicine, Aug;24(7): pp309-12, 1995.

[7] M. Sun, X. Liao, L. Wang, "Cell proliferation in odontogenic jaw cyst epithelium", Hua Xi Kou Qiang Yi Xue Za Zhi, Jun;19(3): pp144-5, 157. 2001

[8] H. Yamamoto, M. Takagi, "Clinicopathologic study of the postoperative maxillary cyst", Oral Surgery Oral Medicine Oral Pathology, Nov;62(5): pp544-8, 1986.

[9] E. Favre-Dauvergne, G. Fleuridas, R. Lockhart, M. Auriol, Y. Le Charpentier, JC Bertrand, F Guilbert. "Paradental cyst", Revue de Stomatologie et Chirurgie Maxillofaciale,97(1): pp7-11, 1996.

[10]R.B. Brannon, “The odontogenic keratocyst. A clinicopathologic study of 312 cases, Part II. Histologic features”. Oral Surgery Oral Medicine Oral Pathology Feb; 43(2):pp233-55,1977.

\section{Author Profile}

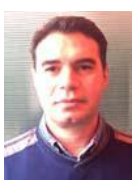

Prof. Dr. Oliver Dimitrovski has graduated at the University "Sts Cyril and Methodius", Faculty of Dentistry in Skopje in 1991/92.He is a specialist of oral surgery since 1998. The degree master of sciense he received in 2003; doctor of science in 2007. Since 2012 he is a professor at the Clinic for Oral Surgery, Faculty of Dentistry in Skopje, R.Macedonia. 